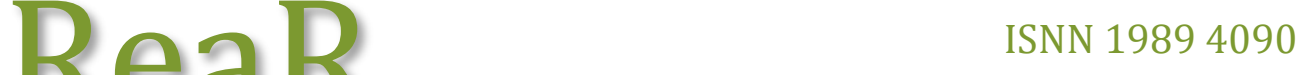 \\ Revista electrónica de AnestesiaR
}

FORMACIÓN MÉDICA

Octubre 2019

\section{¿Es útil el seguimiento de Algoritmos para el manejo de la Vía Aérea Difícil?}

Mariscal Flores M (1), Martínez Hurtado E (2), Rey Tabasco F (2), Castellanos González R (1).

Hospital Universitario de Getafe. Madrid. España

Hospital Universitario Infanta Leonor. Madrid. España.

\section{Resumen}

El seguimiento de los Algoritmos de Vía Aérea Difícil ayuda a conseguir realizar una valoración y planear un manejo de la Vía Aérea de forma sistematizada, para que no se olvide ningún aspecto de la misma.

"Actualmente al no existir un Algoritmo aceptado mundialmente se deben adoptar los mismos al hospital donde se trabaje, a la habilidad de cada uno, a los dispositivos que se tengan y a los pacientes." (Dr. M. Gómez Ríos)

\section{Introducción}

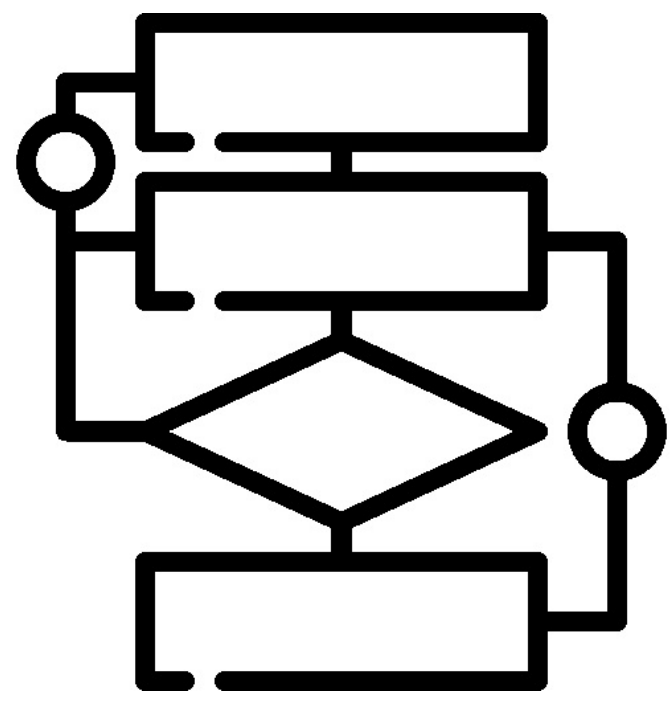

El seguimiento de los Algoritmos de Vía Aérea Difícil ayuda a conseguir realizar una valoración y planear un manejo de la Vía Aérea de forma sistematizada, para que no se olvide ningún aspecto de la misma.

"Actualmente al no existir un Algoritmo aceptado mundialmente se deben adoptar los mismos al hospital donde se trabaje, a la habilidad de cada uno, a los dispositivos que se tengan y a los pacientes." (Dr. M. Gómez Ríos)

os encontramos ante una pregunta que cualquier profesional que maneje la Vía Aérea se ha podido plantear en muchas ocasiones, ya que si dichos algoritmos se miran superficialmente nos pueden llegar confundir.

Es posible distinguir una serie de conceptos con diferencias teóricas que, en la práctica, pueden conllevar a una dificultad para diferenciar dichos términos $\mathrm{y}$, muchas veces, a que se utilicen indistintamente.

- Guías: declaraciones desarrolladas sistemáticamente para ayudar a los profesionales en una circunstancia clínica específica.

- Recomendaciones: estrategias adecuadas $\mathrm{y}$ útiles, no tan estrictas como las guías.

- Protocolos/Algoritmos: procedimientos escalonados $\mathrm{o}$ árboles de decisiones para guiar 
al profesional sobre el diagnóstico y tratamiento de distintos problemas clínicos.

A partir de ahora, en este texto se hablará de Algoritmos, y este término lo podemos definir como "recomendaciones de un grupo de expertos, realizados por consenso, basado en la experiencia, sin una fuerte evidencia científica que no son de obligado cumplimiento y están adaptados al hospital, al operador y al material del que se disponga”.

Si el concepto de Algoritmo lo aplicamos al campo de la Vía Aérea Difícil (VAD), se puede observar que existen una serie de puntos comunes que se repiten en todos los Algoritmos mundiales de VAD, y estos son los siguientes:

- siempre se debe pedir ayuda cuando estemos en una situación de VAD,

- el objetivo final es la oxigenación del paciente independientemente de cómo se consiga, ya sea con ventilación con mascarilla facial o con Dispositivo supraglótico o a través de intubación,

- se debe limitar el número de intentos de intubación,

- se debe considerar la posibilidad en estas circunstancias de despertar al paciente y posponer la cirugía,

- cuando exista una situación "No intubable- no oxigenable", se debe realizar una coniotomía,

- esta decisión puede ser difícil de tomar y a veces se retrasa, lo que lleva a disminuir la seguridad del paciente.

Existe un gran número de Algoritmos de VAD en el mundo, de todos ellos los más conocidos son los de la Sociedad de Americana de Anestesia (ASA) (2) y la Sociedad Británica de VAD (Difficult Airway Society [DAS]) (3).

La ASA describió su primer Algoritmo en 1993, y diez años después lo revisó en 2003, apareciendo en este espacio de tiempo la Mascarilla Laríngea como primera técnica de rescate en situaciones de dificultad para intubar y para ventilar con mascarilla facial.

La última revisión se realizó en 2013 (4), y en este algoritmo se sigue manteniendo la estructura de los dos anteriores, es decir, inicialmente describe una valoración de dificultad de ventilar con mascarilla facial, de introducir un Dispositivo Supraglótico, de realizar una laringoscopia o intubación correcta, o dificultad de realizar una coniotomía en caso necesario.

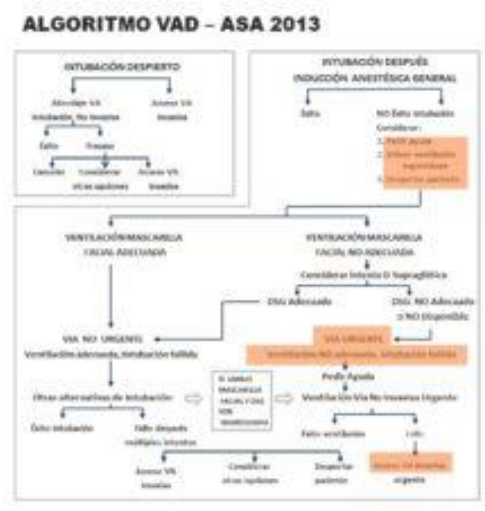

Posteriormente, se establece un plan de manejo de VAD, respondiendo a una serie de preguntas como el realizar la intubación con el paciente despierto o dormido, manteniendo la respiración espontánea o no, utilizando un Videolaringoscopio como primera opción en caso de sospecha de VAD o utilizando una técnica invasiva desde el inicio o no usarla.

Posteriormente, se describe el propio Algoritmo. En un brazo del mismo se plantea la VAD prevista, donde se aconseja realizar la intubación con el paciente despierto, y en el otro brazo la aparición de una VAD no prevista, 
donde se comenta la importancia que el primer intento de intubación sea el "mejor intento o intento óptimo". En este brazo, se recomienda limitar el número de intentos de intubación $\mathrm{y}$ pedir ayuda una vez diagnosticada una VAD.

Una vez que es evidente que no se puede intubar al paciente, se describe si se puede ventilar al paciente con mascarilla facial o no. Si es posible, nos encontramos ante una situación no urgente, $\mathrm{y}$ se aconsejan una serie de dispositivos (fibrobroncoscopio [FBO], videolaringoscopio [VDL], etc.).

Si empieza a fallar la ventilación se comenta el uso de una mascarilla laríngea $\mathrm{y}$, si esta falla, ya nos encontramos en una situación urgente "no intubable- no oxigenable", y se debe valorar el despertar al paciente y posponer la cirugía, y realizar una coniotomía si la situación empeora.

La DAS describe su último algoritmo en 2015 (5). Al igual que el previo de 2004 presenta 4 planes escalonados de manejo de la VAD. Un plan $\mathbf{A}$, en el que se intenta intubar al paciente buscando un intento óptimo con Laringoscopia Directa con ayuda de guías o utilizando como primera $\mathrm{o}$ segunda opción el vídeolaringoscopio.

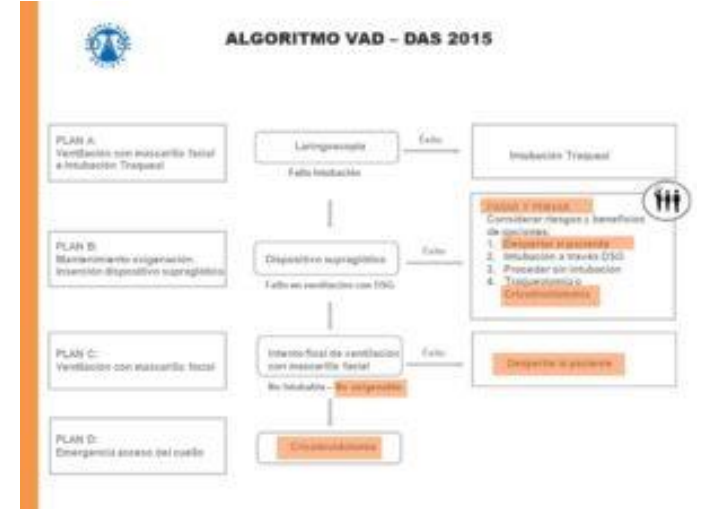

Si éste falla, se pasará al plan B utilizando de rescate un Dispositivo Supraglótico, aconsejando también el uso de la Mascarilla Laríngea de Intubación (ML Fastrach). Se recomienda intubar con ella con la ayuda de un FBO para conseguir un mayor éxito.

$\mathrm{Si}$ no conseguimos la ventilación adecuada se pasará al plan C, intentando de nuevo ventilar con mascarilla facial $\mathrm{y}$, si todo falla $\mathrm{y}$ aparece una situación urgente "no oxigenable- no intubable", se llegará al plan $\mathbf{D}$, en el que se realizará una coniotomía.

En este algoritmo es la primera vez que se menciona el factor humano cuando se comenta, después de fracasar el plan B, "parar y pensar" y hablar con el equipo.

Existen otros muchos Algoritmos como el Canadiense (2,3), que se basa fundamentalmente en tener un buen plan de abordaje y en la comunicación. Los expertos canadienses emitieron una serie de Recomendaciones siguiendo los niveles de evidencia según el protocolo GRADE, diferenciándose 2 partes, una para VAD imprevista y otra para la prevista.

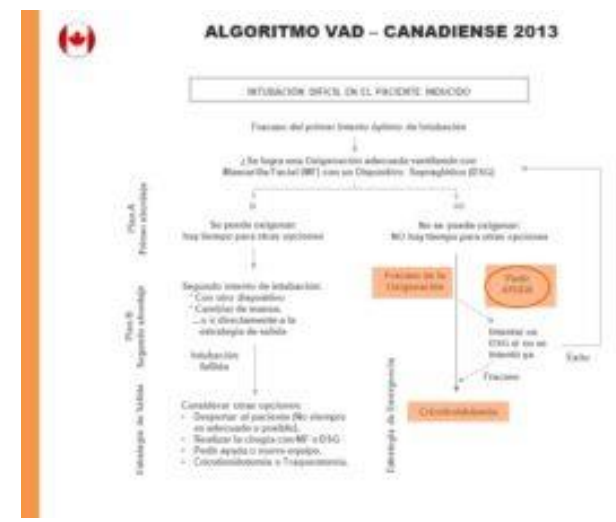

Proponen una serie de diagramas de flujo, mediante los cuales crean un plan de manejo de la VA específico para cada paciente, teniendo en cuenta diversos factores como el número de intentos de intubación previstos por el anestesista, posibilidad de mantener la 
ventilación, cooperación del paciente, experiencia del anestesista, disponibilidad de ayuda...

La sociedad de VAD italiana presenta una serie de Recomendaciones para el manejo de la VAD, dividida en "VAD prevista" y "VAD no prevista" (4). En esta última comienza el diagrama de flujo con PEDIR AYUDA y valorar si la ventilación con mascarilla facial es posible o no. Si la ventilación con mascarilla facial no es posible se recomienda acceso traqueal, mientras que si es posible se permiten máximo 4 intentos de laringoscopia con dispositivos alternativos.

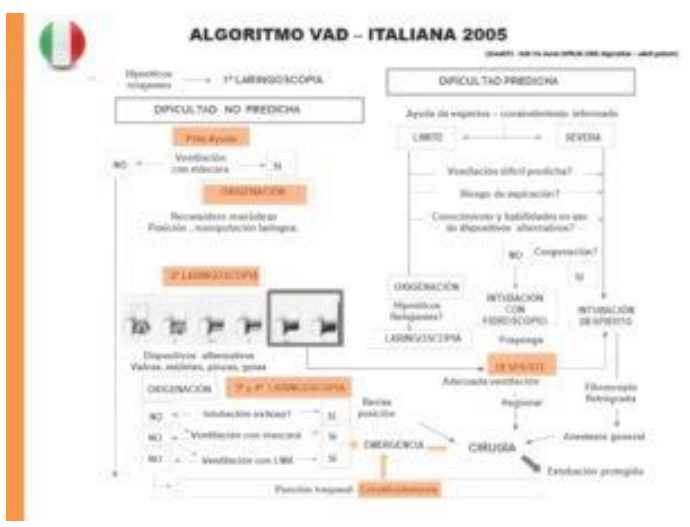

En la VAD prevista diferencia entre VAD "límite" (ventilación con mascarilla facial probablemente con éxito, poco riesgo de aspiración y experiencia con dispositivos alternativos), situación para la que se aconseja inducción con Anestesia General. Y una VAD "severa", donde se recomienda FBO con el paciente despierto.

\section{La sociedad de Anestesia}

Española (SEDAR), a través de un texto de la sociedad Catalana (5), habla de valoración de la VA, manejo de la VA en situaciones específicas como Reanimación, Emergencias, Ventilación unipulmonar, pediatría y obstetricia. Diferencia entre VAD prevista e imprevista, y habla de un plan A, B, C y D. Se permiten 3 intentos de intubación $\mathrm{y}$ un cuarto intento realizado por un experto, y se comenta que la técnica de más éxito es con la que se tenga más habilidad.

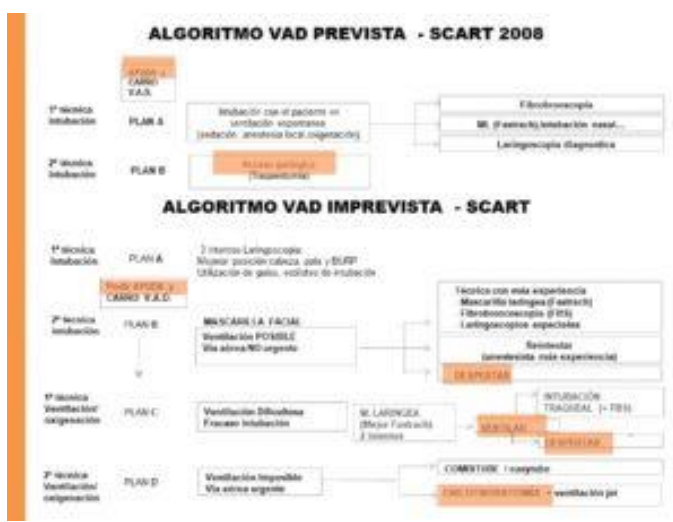

La sociedad India de VAD tiene un gran interés en la enseñanza y entrenamiento en el manejo de la VAD (6). Se habla de 5 algoritmos en situaciones especiales, los 3 primeros referidos a una VAD no prevista en el adulto, en obstetricia y en pediatría, el cuarto sobre problemas encontrados durante la Extubación, y el último referido a manejo de la VAD en UCI. Se hace especial hincapié en la oxigenación.

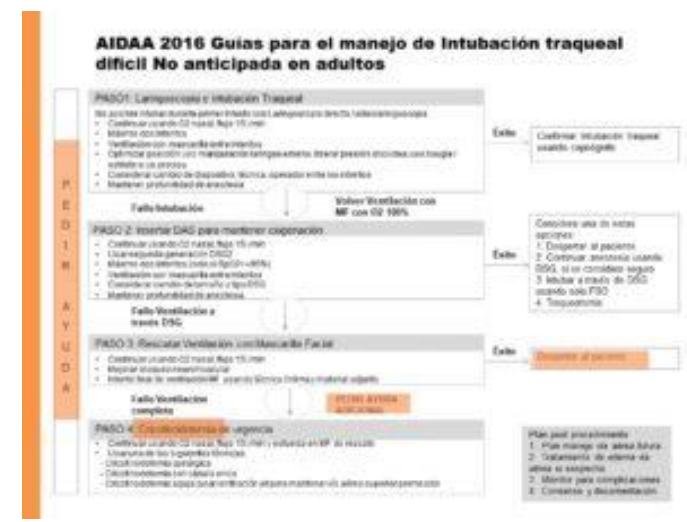

La sociedad Alemana (7) basa su algoritmo en una serie de Recomendaciones de distinto grado $\mathrm{y}$ unos diagramas de flujos para la VAD prevista o no prevista y, al final, da unos requerimientos principales como son: evaluación de la VA, requerimientos mínimos en un quirófano para el manejo de la VA (ventilación con mascarilla facial, dispositivo supraglótico, ayuda a la laringoscopia directa, 
videolaringoscopios, set de coniotomía o traqueostomía).

Si hay evidencia de ventilación con mascarilla facial imposible o intubación mediante laringoscopia directa, se recomienda intubar con respiración espontánea o FBO con el paciente despierto. Aconsejan usar mascarillas laríngeas de $2^{\mathrm{a}}$ Generación, no realizar más de 2 intentos, y destacan el importante papel de los videolaringoscopios. $\mathrm{Si}$ falla la intubación y la oxigenación, recomiendan realizar una coniotomía.

Existirá siempre una estrategia planeada para la extubación. Y se destaca la gran importancia del entrenamiento y la Docencia del manejo de la VAD, así como el disponer de unos Algoritmos adaptados a los escenarios clínicos particulares disponibles.

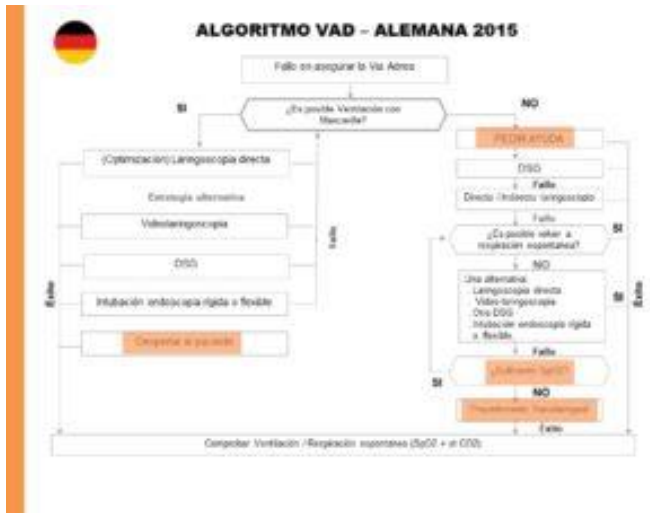

La sociedad Japonesa (8) presenta, en nuestra opinión, unas guías de gran originalidad. Definen 3 grados de ventilación usando la onda del capnograma:

- $\quad \mathrm{V} 1$, en donde se identifican las 3 fases del capnograma, y se correspondería con una ventilación normal.

- V2, en donde solo se ve la fase II (rápido ascenso), siendo en esta situación la ventilación ineficaz.
- V3, en donde existe ausencia de onda y solo se observa una línea basal, que indica una ventilación anormal, ya sea por hipoventilación o apnea.

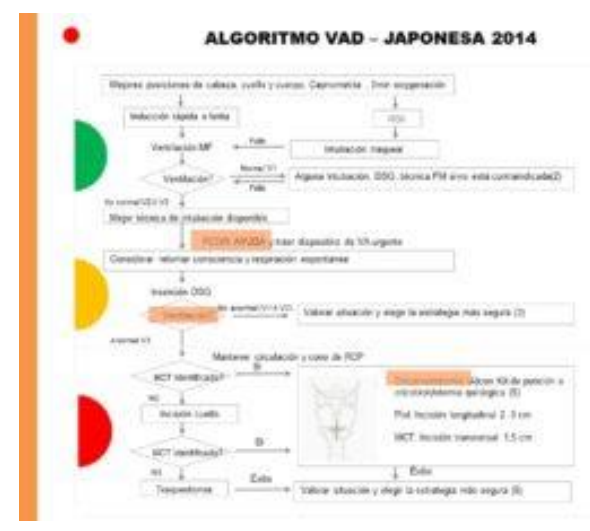

Se definen 3 zonas distintas de 3 colores (verde amarillo y rojo) (como los colores de un semáforo), dependiendo del grado de riesgo del paciente y la graduación de la ventilación:

- zona verde (V1), condición segura,

- amarilla semi-emergencia (V2),

- y roja emergencia crítica (V3).

$\mathrm{Si}$ se estudian en profundidad los algoritmos de VA (como así lo hizo un grupo de anestesiólogos hispano hablantes liderado por el anestesiólogo Dr. Garzón), se puede observar una serie de recomendaciones básicas que se dividen en varios sectores:

\section{Definición.}

2. Terminología precisa.

3. Valoración preanestésica.

4. Evaluación preoperatoria de la VA debe ser sistemática. (suma de los tests).

5. Muy importante la cooperación del paciente.

6. Preparación. Material. Preoxigenación.

7. Planificación, preparación $y$ comunicación cuidadosa del procedimiento a seguir. 
8. Preoxigenación. Fracción expirada de oxígeno $>\quad 90 \%$. Técnicas de oxigenación apnéica.

9. "Posición de olfateo" y "posición de rampa".

10. VAD prevista.

11. Información al paciente de su procedimiento de intubación despierto.

12. Transmitir confianza y tranquilidad.

13. FBO despierto o VDL despierto (Plan A).

14. Vía quirúrgica (Plan B).

15. VAD no prevista.

16. Optimización primer intento de intubación.

17. $\mathrm{N}^{\mathrm{o}}$ limitados de intentos.

18. Oxigenación es el objetivo.

19. Relajación muscular antes de ventilación con MF.

20. DSG de $2^{\text {a }}$ Generación

21. "Situación no intubable- no oxigenable": despertar y volver a la ventilación espontánea. $\mathrm{Si}$ no es posible realizar coniotomía.

22. Extubación.

23. Los pacientes con VAD en la intubación, reevaluación antes de la Extubación.

24. Posponer la Extubación si no hay seguridad.

25. Intercambiador/DSG.

\section{Educación en vía aérea.}

- Formación continuada habilidades técnicas y no técnicas. Simulación de alta fidelidad.

- Rotación VAD para residentes.

\section{Registro y notificación en VAD.}

- Notificación por escrito/verbal. Tarjeta VAD.

- Registro en la historia.
Con todo lo anteriormente mencionado, se puede responder a la pregunta que da título a este texto " $¿ E s$ útil el seguimiento de algoritmos para el manejo de la Vía Aérea Difícil? Y la respuesta sería SI.

Con el seguimiento de los algoritmos se conseguiría una sistematización en el anejo de la VAD.

No obstante, hay una serie de aspectos que se deben mejorar, antes de nada, como es todo aquello que se relacione con el Factor Humano (trabajo en equipo, liderazgo, comunicación...), para lo que es muy importante trabajar con Simulación de alta fidelidad y establecer en las residencias de Anestesiología una rotación reglada por Vía Aérea Difícil.

Podemos concluir con las palabras del Dr. Gómez Ríos (9):

"En la ausencia de una guía universalmente aceptada, se adaptarán los algoritmos a competencias especificas, juicio individual, disponibilidad, habilidad con dispositivos y características del paciente".

\section{Bibliografía}

1.Presentación Grupo de Vía Aérea Hispanohablante (GVAH). AnestesiaR

2. Apfelbaum JL, HagbergCA, Caplan RA, et al. Practice guidelines for management of the difficult airway: an updated report by the American Society of Anesthesiologists Task Force on Management of the Difficult Airway. Anesthesiology. 2013;118:251270. (ubMed)

3.Frerk C, Mitchell VS, McNarry AF, Mendonca C, Bhagrath R, Patel A, O'Sullivan EP, Woodall NM, Ahmad I. Difficult Airway Society intubation guidelines working group. Difficult Airway Society 2015 guidelines for management of unanticipated difficult intubation in adults. Br J Anaesth. 2015 Nov 10. 
pii: aev371. [Epub ahead of print] ( (pdf) (web)

4.Resumen del Algoritmo Vía Aérea Difícil de la American Society of Anesthesiologist 2013. AnestesiaR

5.Resumen de las Guías de Intubación Difícil Imprevista DAS 2015. AnestesiaR
Correspondencia al autor

\section{Marisa Mariscal Flores}

mmariscalflores@gmail.com

Jefa de Sección. Servicio de Anestesia y Reanimación.

Hospital Universitario de Getafe. Madrid. España.

Grupo de vía aérea de SAR Madrid. Grupo de Via Aérea Hispanohablante (GVAH).

Aceptado para el blog en mayo de 2019. 